\title{
Template Matching Recognition Algorithm Based On Grid Characteristics
}

\author{
Besse Magnim $^{1}$ and Song $\mathrm{Kai}^{2}$ \\ ${ }^{1}$ Electronic and Communication Engineering \\ Shenyang Polytechnic University, Liaoning Nan Hu 6 road/China \\ ${ }^{2}$ Information and Communication Engineering \\ Shenyang Polytechnic University, Liaoning Nan Hu 6 road/China \\ ${ }^{1}$ magnimbesse@gmail.com; ${ }^{2}$ ap9351@163.com
}

\begin{abstract}
In order to identify the license plate characters accurately, put forward a kind of template matching recognition algorithm based on grid characteristics. This algorithm by comparing the grid characteristics of extracted template and characters to be recognized, to realize the recognition of license plate characters. First of all, 34 templates characters can be divided into 12 areas and the grid characteristics of each template can be extracted, respectively. Then, divide the characters, which should be recognized, into 12 areas and extract the characteristic. Finally, compare the grid characteristics of each area that between the templates and to identify the characters, calculate the weighted sum. The minimum weighted sums of the corresponding template is the result of identification. The experimental results show that the method can effectively reduce the number of templates and unnecessary matching process, as well as to improve the recognition speed and accuracy.
\end{abstract}

Keywords: grid characteristic, template matching, character recognition

\section{Introduction}

Template matching is a technique in computer vision used for finding a sub-image of a target image which matches a template image. This technique is widely used in object detection fields such as vehicle tracking, robotics, medical imaging, and manufacturing.

The crucial is to adopt an appropriate measure to quantify similarity or matching. However, this method also requires aextensive computational cost since the matching process involves moving the template image to all possible positions in a larger target image and computing a numerical index that indicates how well the template matches the image in that position. This problem is thus considered as an optimization problem.

A reasonable first step to approaching such a task is to define a measure or a cost measuring the distance or the similarity between the known reference patterns and the unknown test pattern, in order to perform the matching operation known as template matching.

The research on "License plate recognition technology" is also an application of Artificial Intelligence Technology. With a view to attain higher intelligent traffic management level, it introduces computer information management technology, digital photography, as well as selected advanced image processing, pattern recognition and artificial intelligence. License plate recognition technology consists of three significant components: positioning of vehicle license plates, segmentation of license plate characters and recognition of license plate characters. Among them, character recognition is a method that is based on template matching and neural network.[1] For template matching recognition algorithm, if the lighting changes, the test plate characters are not clear or the size changes, the template matching accuracy rate will be impacted.[2] Thus, we use a multiple templates or large template for matching to

Received (June 9, 2017), Review Result (August 17, 2017), Accepted (September 21, 2017) 
improve accuracy rate. However, the processing speed will slow with the increase of the number of template or the area of template. [3] For Neural network recognition algorithm, its recognition effect is relevant to the features that are extracted from characters. But, the extraction of character features is time-consuming. Thus, it becomes the key to character recognition. [4].

\section{The Summary of License Plate Recognition}

\subsection{Character Recognition}

License plate recognition system is divided into three parts, [5] License plate locating, character segmentation and character recognition. Among them, character recognition is the last link. This link will decide whether the front link's efforts can get satisfactory result. The flow chart of license plate recognition is as shown in the Figure 1:

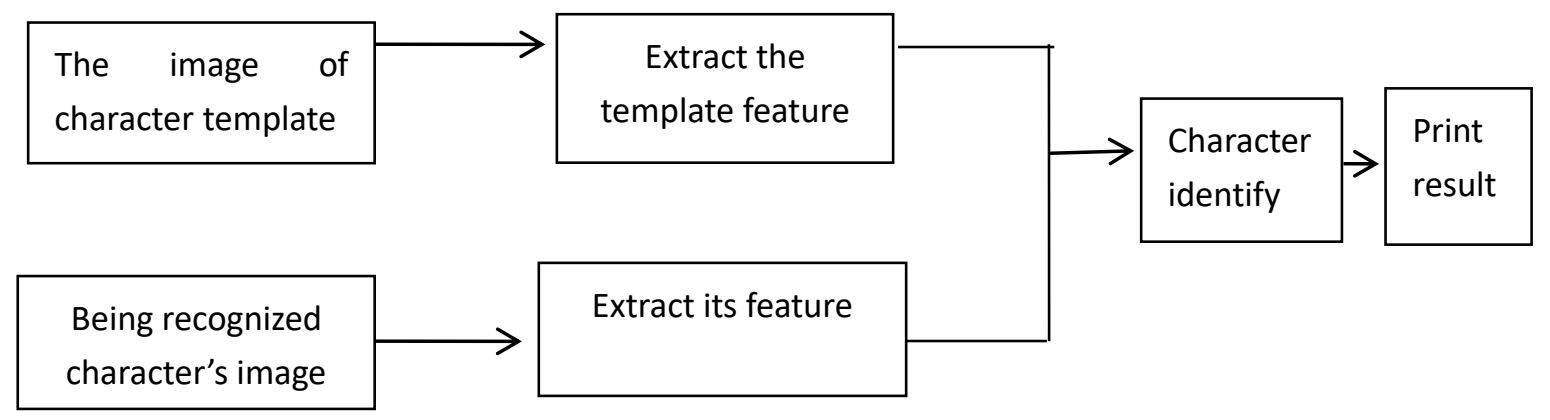

Figure 1. The Flow Chart of License Plate Recognition

Before starting with the character recognition algorithm study, first it is necessary to know about the situation of our country's license plate characters.

\subsection{The Prior Knowledge of License Plate Character}

1)The first character of the character set is the Chinese character set that contains the short for provinces and cities, such as a, b, c, d and p, etc.;

2) The second character of the character set is English alphabetic character set that contains A, B, C and others, in total 26 English letters;

3) The third character to the seventh character of the character set consists of A, B, C and other 26 English letters and 10 Arabic numeral characters from 0 to 9, a total of 36 elements;

Above all, the largest sample set of license plat recognition is the fifth character from the 36 character elements. So the character recognition belongs to pattern recognition, which is based on small sample standard character set.[6]

At present, both at home and abroad, the character recognition usually uses the neural network or template matching method. Because the automobile license plate characters of our country are of a normative and rather small nature, the system uses relatively simple template matching method.

\section{License Plate Character Feature Extraction}

Due to the customized form of the license plate specifications, the license plate characters also has specification and are easy in application for extracting features. There are many methods to extract character feature. For instance, it is possible to use the projection method to put the whole character's image into projection, use the values as this character's feature. Besides, you also can extract the features of character image's each part, and then put all extraction features together to form the characteristics of the character.

As shown, the Figure 2 is a method schematic diagram that extracts grid feature. Among them, the Figure 2(a) is a size of $20 * 40$ template character A, the Figure 2(b) equally divides 
character into four small templates from the transverse and longitudinal, the Figure 2(c) divides it just in the transverse, and the Figure 2(d) divides it just in the longitudinal.

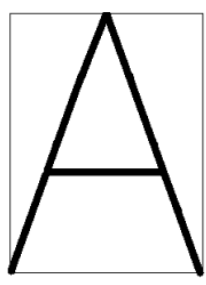

(a)

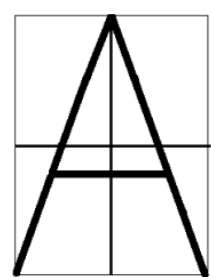

(b)

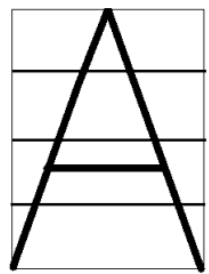

(c)

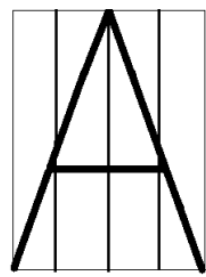

(d)

Figure 2. The Template Image Feature Extraction

The main idea of the grid feature extract to character template is:

First, equally divide character image into 4 large grids: a1, a2, a3,a4, statistic the number of black pixels in each grid to form a 4 dimensional vector;

Second, in the vertical direction of the quartered place in order to create the horizontal line, it was divided into four areas: a5, a6, a7, a8 statistic the number of black pixels in four areas; a4 dimensional vector was also formed;

Similarly, in the horizontal direction of the quartered place in order to create the vertical line, it was divided into four areas: a9, a10, a10, a12 statistic the number of black pixels in four areas; a4 dimensional vector was also formed;

According to the above method, statistic the number of each character's 12 dimensional pixels and save it to template feature library, and the feature library file named template data that stored in the data folder.

For example, the 12 dimensional feature extraction space vector of the template $\mathrm{A}$ is (a1, a2... a12), the form is as follows:

Table 1. The Feature Vector of Template A

\begin{tabular}{|c|c|c|c|c|c|c|}
\hline A & a1 & $\mathrm{a} 2$ & a3 & $\ldots$ & a11 & a12 \\
\hline $\begin{array}{ll}\text { Range } & \text { of } \\
\text { column } & \\
\text { coordinates } & \end{array}$ & $0-9$ & $9-19$ & $0-9$ & $\ldots$ & $9-14$ & 14-19 \\
\hline $\begin{array}{l}\text { Range of Line } \\
\text { coordinates }\end{array}$ & $0-19$ & $0-19$ & $19-39$ & $\ldots$ & - & - \\
\hline number & numa1 & numa2 & numa3 & $\ldots$ & numa11 & numa12 \\
\hline
\end{tabular}

Similarly, in accordance with the above method recorded, Figure 3 shows the characteristic dimension of the missing characters in the region (a1, a2, a4, a5, a6, a7, a8, a10, a11, a12,).
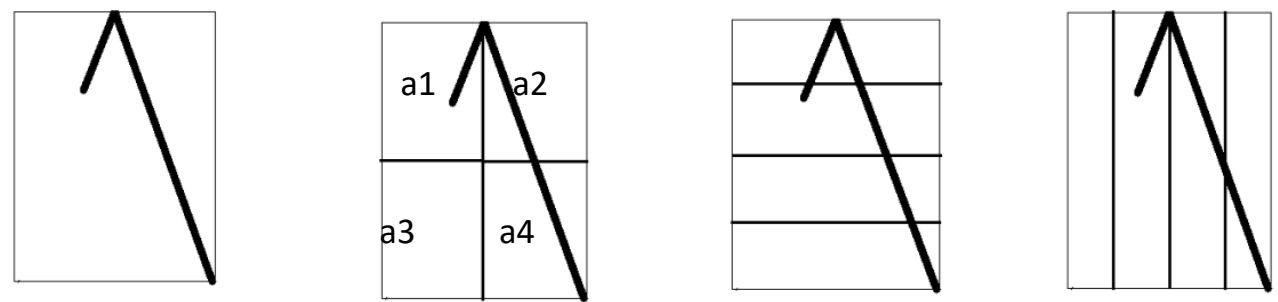

Figure 3. The Feature Extraction Image of Incomplete Character

When the horizontal or vertical line coincides with a horizontal or vertical line of 
characters, sharing the number of pixels in the corresponding region is not recorded, that is zero. For example, the characters $\mathrm{T}$ and $\mathrm{Y}$ are without pixels in the area of $\mathrm{a} 3$ and $\mathrm{a} 4$, the area ID is only a1 and a2, as shown in Figure 4. Figure 3, the incomplete character area contains a4 area, so there is no need to compare or recognize with template character $\mathrm{Y}$ and $\mathrm{T}$, which improve recognition speed and reduce the rate of miscarriage of justice.

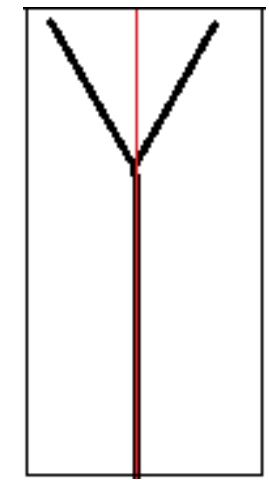

(a) The character $Y$ and vertical line overlap

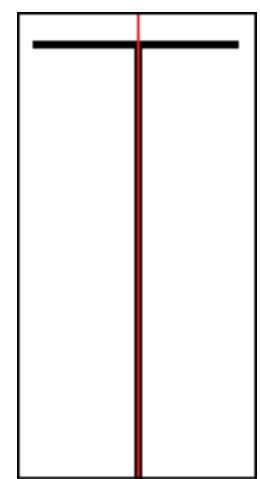

(b) The character T and vertical line overlap

Figure 4. The Example Graph of Vertical and Vertical Distribution Character Overlap

\section{License Plate Character Recognition}

License plate recognition involves capturing photographic video or images of license plates; whereby they are processed by a series of algorithms that are able to provide an alpha numeric conversion of the captured license plates images into a text entry.

When identifying characters, first, compare abstracted character image that will be identified with template image, and then conclude which template feature matches similarity higher, thereby, determine those characters to be identified.

Since the strokes of Chinese character are abundant and Chinese characters only appear at the first character of the license plate, we process Chinese characters and other characters separately - Chinese characters only match with characters, which are in the Chinese character sample database. This article only deals with number and letter identification.

\subsection{The Principle of Template Matching}

For templates, without strong features, or for when the bulk of the template image constitutes the matching image, a template based approach may be effective. As aforementioned, since template -based template matching may potentially require sampling of a large number of points; it is impossible to reduce the number of sampling points by reducing the resolution of search and templates images by the same factor and performing the operation on the resultant downsized images.

In that case we can say, Template matching is a process of comparing known template with part of being tested zone, which has the same size. First, we make the top left point of template coincide with the first pixel of the image. Then, we move the template to next pixel. We continue operating in that way until all the pixels have been compared. At that time, part of zone, which has the minimum degree of difference, is what we are looking for.

The principle of template matching is as follows:

A template $T(M \times N)$ stacked on the search graph $S(W \times H)$ to translation, the area covered by the template called sub graph $S_{i j}, \mathrm{i}, \mathrm{j}$ is the upper left corner pixel coordinates of this diagram in $\mathrm{S}$, called the reference point. The search scope is: 


$$
\begin{aligned}
& 1 \leq i \leq W-N+1 \\
& 1 \leq j \leq H-M+1
\end{aligned}
$$

The similarity relation function is:

$$
D(i, j)=\sum_{m=1}^{M} \sum_{n=1}^{N}\left[S_{i j}(m, n)-T(m, n)\right]^{2}
$$

Expand the formula (3-2), inferred:

$$
D(i, j)=\sum_{m=1}^{M} \sum_{n=1}^{N}\left[S_{i j}(m, n)\right]^{2}-2 \sum_{m=1}^{M} \sum_{n=1}^{N}\left[S_{i j}(m, n)\right] \times T(m, n)+\sum_{m=1}^{M} \sum_{n=1}^{N}[T(m, n)]^{2}
$$

The interpretation of the formula (3) is: The first item is the energy of the sub graph covered by the template, the third item is the total energy of the template and they are independent of the template matching. The second item is the relationship between the template and the sub graph. Along with $(i, j)$ is changed to change. When the template $T$ and sub graph $S_{i j}$ match, this item obtain the maximum value[9]. Therefore, the function can be defined as:

$$
R(i, j)=\frac{\sum_{m=1}^{M} \sum_{n=1}^{N} S_{i j}(m, n) \times T(m, n)}{\sum_{m=1}^{M} \sum_{n=1}^{N}\left[S_{i j}(m, n)\right]^{2}}
$$

Normalization:

$$
R(i, j)=\frac{\sum_{m=1}^{M} \sum_{n=1}^{N} S_{i j}(m, n) \times T(m, n)}{\sqrt{\sum_{m=1}^{M} \sum_{n=1}^{N}\left[S_{i j}(m, n)\right]^{2}} \sqrt{\sum_{m=1}^{M} \sum_{n=1}^{N}[T(m, n)]^{2}}}
$$

When the template is exactly the same as the sub graph, the correlation coefficient $R(i, j)=1$; otherwise, $\mathrm{R}(\mathrm{i}, \mathrm{j})<1$. Therefore, the correlation coefficient is bigger, when the template and the search graph are more similar. You can find out the maximum value of the correlation coefficient $R_{\max }(i, j)$ after all searches are done in the searched graph. Then the corresponding sub graph $S_{i j}$ is the matching target.

\subsection{The Improved Template Matching Method}

The traditional template matching recognition method needs to match point by point, in which the computation is long and the speed is too slow. The matching speed of big templates often are too slow; and the matching speed of small template is, the faster is the matching speed.

According to the grid features extracted from the above (Figure 3), calculate the weighted distance between it and the feature vectors of each template. The minimum weighted distance is the recognition result, which is the character recognition. Compare with the extracted feature area of the character, which is to be recognized and the template corresponding areas, that is the $(\mathrm{a} 1, \mathrm{a} 2, \mathrm{a} 4, \mathrm{a} 5, \mathrm{a} 6, \mathrm{a} 7, \mathrm{a} 8, \mathrm{a} 10, \mathrm{a} 11, \mathrm{a} 12)$ region, measure the error between $\mathrm{T}$ and $S_{i j}$,the formula is as follows:

$$
D(i, j)=\sum_{m=1}^{M} \sum_{n=1}^{N}\left|S_{i j}(m, n)-T(m, n)\right|
$$


Among them, when $D(i, j)$ get the minimum, that is the matching target. This method can reduce the unnecessary computation quantity only by comparing the area, which is extracted from the recognition character. In Figure 3, the characteristic region of the missing letter don't have $\mathrm{A} 3$ and $\mathrm{A} 9$ regions.

Process is as follows:

Divide template character into four small templates and mark them as "a1, a2, a3, a4". Similarly, the character image to be recognized is divided into four zones as well;

Match small template "a1" to characters zone "al" - which will be tested, and calculate the degree of matching difference "d1";

Similarly, work out "d2","d3","d4";

Sum up the degree of difference:

$$
D 1=\sum_{i=1}^{4} d i
$$

Abstract 34 templates four zone successively and match them with the corresponding zone of character images, which will be identified soon. Calculate the cumulative degree of difference between each template with characters to be identified - "Dj", take the minimum. The corresponding template of $(i, j)$ is exactly the recognition result.

After the completion of a character recognition, extract the next character and recognize it, until the completion of recognition for the segmentation of all characters. The flow chart is shown in Figure 5:

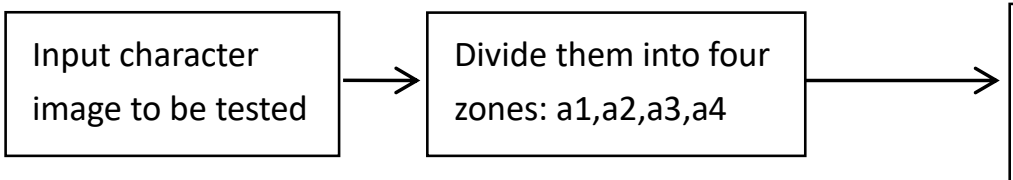

Abstract the first template's a1 zone and match it with character a1 sequentially, then calculate the difference degree $\mathrm{d} 1$

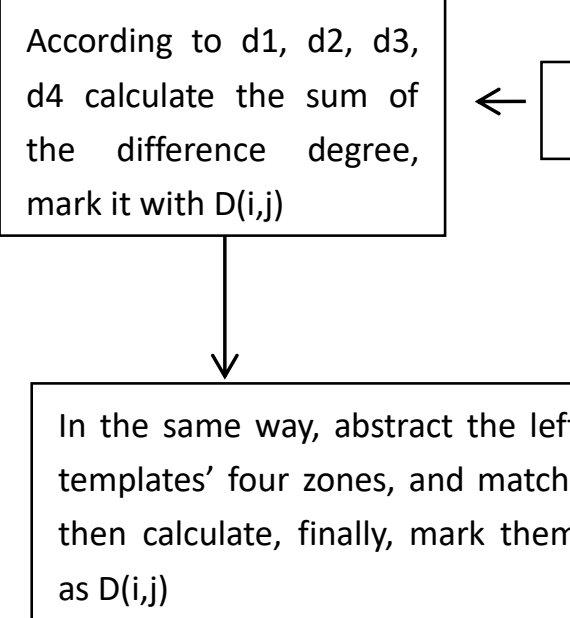

Figure 5. Flow Chart of Template Matching

\section{Conclusion}

According to our work, whose concern License plate recognition system; the main of the algorithm we proposed is to have successive subdivisions to the license plate. The first step 
was to subdivide it in to 34 separates templates; after that every small parts in to 12 templates. Therefore templates quantity decrease and the recognition speed will increased. The method is to privileged the template whose contain characters pixels. Our algorithm results are more effective than filtering process because of the speed of recognition and the accuracy rate.

Our work can be opened to radiology image accuracy rate or in meteorology.

\section{References}

[1] B. H. Ma, L. Chen and L. Liang, "Application of real time and Fu Liye transform correlation recognition in license plate character recognition”, Journal of Guangdong University of Technology, vol. 26, no. 3, (2009), pp. 68-71.

[2] W. Gang, "Research on vehicle detection and license plate recognition algorithm", Aiyuan University of Technology, (2009).

[3] T. Sirithinaphong and K. Chamnongthai, "Extracting of Car License Plate Using Motor Vehicle Regulation and Character Pattern Recognition", IEEE Asia -Pacific Conference on Circuits and System s - Proceed2ings, (1998), pp. 559- 562.

[4] N. Xu, "Research and design of automatic detection and recognition system for traffic violation", Shandong University, (2006).

[5] J. Shen, H. Liu and C. Zhou, "The classic cases detailed about Visual C++ digital image processing", Beijing: Machinery Industry Press, vol. 6, (2012), pp. 397,434-435,439-441.

[6] W. Feng, H. Liang and C. Wang, "The classic cases detailed about Visual C++ digital image pattern recognition”, Beijing: Machinery Industry Press, vol. 6, (2012), pp. 392-395,428-430,476.

[7] H. Zhang, "Proficient in C++ Visual digital image pattern recognition technology and engineering practice", 2edition.Beijing : People's Posts and Telecommunicationa Press, vol. 8, (2008), pp. 206-219,397-399,413-416.

[8] B.-F. Wu, S.-P. Lin and C.-Chen, "Extracting characters from real vehicle license Plates out-of-doors", IET Computer \& Vision, vol. 1, no. 1, (2007), pp. 2-10.

[9] Y. R. Huang and H. Duan, "Edge detection of license Plate based on wavelet transform and quantum genetic algorithm", Proceedings of the SPIE on Automatic Target Recognition, Image Analysis and Multispectral Image Acquisition, (2007), pp. 6786-6789. 
International Journal of Signal Processing, Image Processing and Pattern Recognition Vol. 10, No. 10 (2017) 